Journal of Bangladesh Academy of Sciences, Vol. 36, No. 1, 123-135, 2012

\title{
STOKES FLOW BETWEEN TWO CYLINDERS
}

\author{
A. MURAD* AND S. K. SEN ${ }^{1}$ \\ Department of Mathematics, University of Chittagong, Chittagong-4331, Bangladesh
}

\begin{abstract}
The two-dimensional slow viscous fluid motion between two co-axial circular cylinders showed the inner cylinder is shear-free and the outer one is rigid. The flow is due to the presence of a line source and a line sink of equal strength on the outer cylinder. The stream function for the flow in the annular region is established. The hydrodynamic force on the inner shear-free cylinder has been evaluated. Some numerical values for the force have been presented in a table and compared with corresponding known values where both inner and outer cylinders are rigid.
\end{abstract}

Key words: Stokes flow, Line source, Line sink, Co-axial cylinders, Shear-free, Stream function

\section{INTRODUCTION}

Rayleigh (1893) studied the two-dimensional Stokes flow within a single circular cylinder due to a line source and a line sink of equal strength on the circular boundary situated at opposite ends of a diameter. The model can be thought of as fluid enters into the cylinder through one small perforation and leaves the enclosure by another one. Ranger (1961) examined the case of two-dimensional slow viscous fluid motion in the annular region of co-axial circular cylinders where a line source and a line sink of equal strength are placed at arbitrary points on outer cylinder. Recently, Chen (2000) investigated the plane Stokes flow in the annular junction formed by co-axial cylinders, due to one line source and two line sinks, noting that the sum of the strengths of the sinks is equal to that of the source in magnitude; and they all lie on the outer boundary. In all the three cases mentioned above no-slip boundary condition, i.e. fluids adhere to boundaries, were considered. The present authors were interested in studying slow viscous fluid flow in the annular region between coaxial rigid and shear-stress-free (i.e. frictionless) circular boundaries.

Usha and Hemalatha (1993) studied the problem of two-dimensional slow viscous fluid motion around a shear-stress-free circular cylinder; they contributed a circle theorem for plane Stokes flow past an impermeable and shear-stress-free (also termed shear-free or free surface) circular cylinder, showing a model for two phase fluid system.

\footnotetext{
* Corresponding author: <ammathcul@yahoo.com>.

${ }^{1}$ Research Centre for Mathematical and Physical Sciences (RCMPS), University of Chittagong, Chittagong-4331, Bangladesh.
} 
In this study Usha and Hemalatha (1993), pointed out that shear-stress-free boundary conditions arise naturally in various fields of interest in technology and geophysics. Again for a three-dimensional situation Harper (1983) considered shear-stress-free spherical boundaries and formulated a general theorem for axisymmetric viscous flow past a shear-free sphere. He cited that the earth's core is, to a good approximation, a shear-stress-free boundary for the convection in its mantle. Planiappan et al. (1996) examined the problem of axisymmetric Stokes flow around a shear-free double-body and extended Harper's sphere theorem (Harper 1983) to flow past a shear-free double-body consisting of two unequal, orthogonally intersecting spheres. In this context, a theorem for shear-free sphere in Stokes flow due to Palaniappan et al. (1990) is also noteworthy. The authors noted that the surface of a clean bubble is a shear-free boundary for the flow outside it in an uncontaminated viscous fluid (Daripa and Palaniappan 2001, FigueroaEspinoza et al. 2008, Harper 1983, Legendre et al. 2003, Palaniappan 1996, Usha and Hemalatha 1993). Daripa and Palaniappan (2001) examined the two-dimensional Stokes flow past a shear-free double circle (a two-dimensional stationary composite bubble) and presented a theorem to calculate to disturbed flow when a shear-free double circle is inserted into a given unbounded slow viscous fluid motion. In the same paper (Daripa and Palaniappan 2001), for three-dimensional situation, they formulated a theorem to evaluate the perturbed flow when a shear-free double sphere (a composite bubble) is introduced into an arbitrary axisymmetric unbounded Stokes flow. Here, it is important to note that the circle theorem or the sphere theorems are respectively for the flow fields due to the fundamental singularities outside (or, inside) a shear-free circular boundary or spherical boundary. Happel and Brenner (1986) investigated creeping motion of a rigid sphere at the centre of an outer shear-free spherical envelope. Happel and Ast (Happel and Brenner 1986) studied the axial creeping motion of a rigid sphere within a shear-free cylinder, a sphere-in-cylinder type of cell model. Again, Happel (Happel and Brenner 1986) examined the slow viscous fluid motion in the annular region formed by two concentric circular cylinders where the inner cylinder is rigid and the outer one is a shearfree (frictionless) cylindrical envelope, a cylinder-in cylinder type of cell model, for the cases: (1) flow is assumed to be parallel to axes of the cylinders, and (2) the flow is at right angles to the axes.

In the present paper the objective was to study two-dimensional slow viscous incompressible fluid motion in the annular region formed by an inner circular shear-free cylinder and a co-axial outer circular rigid cylinder and where the flow is due to the presence of a line source and line sink of equal strength on the outer cylinder. This model may be thought of as follows: Suppose that a two-dimensional circular clean bubble is concentrically placed inside a rigid circular boundary. Authors are interested in studying the two-dimensional Stokes flow around the two-dimensional circular clean bubble where 
the flow is due to the reason that a viscous fluid enters and leaves the enclousure though two small perforations on the outer cylinder. Equivalently, the model can also be thought of as follows. Suppose that there is a fluid cylinder of vanishing viscosity in a co-axial position with the outer rigid cylinder; a viscous fluid enters and leaves the annular region by means of two small perforations on the outer cylinder.

Here the main interest is to calculate the stream function for the flow mentioned above. Furthermore, the hydrodynamic force and torque exerted by the moving fluid on the inner shear-free cylinder are evaluated.

\section{MATHEMATICAL FORMULATION AND SOLUTION OF THE PROBLEM}

(a) Flow inside a rigid circular boundary: At the beginning we derive the stream function for the slow viscous fluid motion within a fixed rigid circular cylinder of radius $a$, due to a line source of strength $m$ and a line sink of equal strength situated at arbitrary points on the cylinder. Here it is convenient to take the centre of the circle at the origin of polar co-ordinates $(r, \theta)$, and the co-ordinates of the source and sink are $A(a, \alpha)$ and $B(a,-$ $\alpha$ ), respectively (Fig. 1).

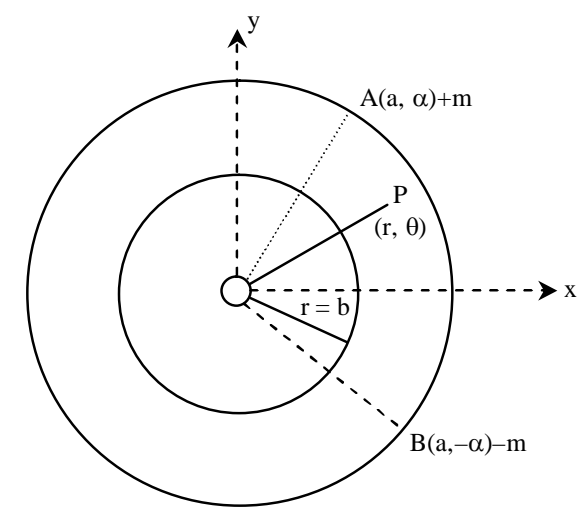

Fig. 1. Definition sketch of flow between co-axial cylinders where the inner one is shear-stress-free and the outer one is rigid.

The stream function for the combination of the source and sink of equal strengths $m$ alone is evidently

$$
\psi_{0}=m\left\{\tan ^{-1}\left(\frac{r \sin \theta-a \sin \alpha}{r \cos \theta-a \cos \alpha}\right)-\tan ^{-1}\left(\frac{r \sin \theta+a \sin \alpha}{r \cos \theta-a \cos \alpha}\right)\right\}
$$

Clearly, the circle $r=a$ is a streamline, except for the points of discontinuity $A(a, \alpha)$ and $\mathrm{B}(a,-\alpha)$ on it, where the flow is related to the source and sink. The stream function in equation (1), for $r \leq a$, may be expanded in the following form 
$\psi_{0}=-2 m \alpha-2 m \sum_{n=1}^{\infty} \frac{1}{n}\left(\frac{r}{a}\right)^{n} \cos n \theta \sin n \alpha$

Now, we introduce a rigid circular cylinder $r=a$, and the resulting flow may be represented by

$\psi_{1}=\psi_{0}+\psi^{*}$,

where $\psi_{0}^{*}$ is the perturbation stream function.

$\nabla^{4} \psi_{1}=0$

where $\nabla^{2}=\left(\frac{\partial^{2}}{\partial r^{2}}+\frac{1}{r} \frac{\partial}{\partial r}+\frac{1}{r^{2}} \frac{\partial^{2}}{\partial \theta^{2}}\right)$

and conditions on the boundary

$\psi_{1}(r, \theta)=0 \quad$ on $\quad r=a$,

$\frac{\partial \psi_{1}}{\partial r}(r, \theta)=0 \quad$ on $\quad r=a$.

Again $\psi_{0}^{*}(r, \theta)$ satisfies the bi-harmonic equation

$\nabla^{4} \psi_{0}^{*}=0$,

and boundary conditions

$$
\begin{aligned}
& \psi_{0}^{*}(r, \theta)=\psi_{0}(r, \theta) \quad \text { on } \quad r=a, \\
& \frac{\psi_{0}^{*}}{\partial r}(r, \theta)=-\frac{\partial \psi_{0}}{\partial r}(r, \theta) \quad \text { on } \quad r=a .
\end{aligned}
$$

The components of the hydrodynamic velocity $\left(u_{r}, u_{\theta}\right)$ with reference to plane polar coordinates $(r, \theta)$ are given by

$u_{r}=\frac{1}{r} \frac{\partial \psi_{1}}{\partial \theta}, u_{\theta}=-\frac{\partial \psi_{1}}{\partial \theta}$

both of which vanish at every point on the boudary $r=a$ except at the source $A$ and $\operatorname{sink} B$.

Using the circle theorem (Appendix) we now derive the stream function of the resulting flow inside a rigid circular cylinder of radius $a$ due to a line source and a line sink of equal strengths $\mathrm{m}$ situated at points $\mathrm{A}(a, \alpha)$ and $\mathrm{B}(a,-\alpha)$ on the cylinder. Since the stream function (1) satisfies the condition $\psi_{0} \sim 0\left(r^{k}\right), k>1$, by applying the theorem, the stream function for the new flow within the circular boundary becomes 


$$
\begin{gathered}
\psi_{1}(r, \theta)=-m\left\{\tan ^{-1}\left(\frac{r \sin \theta-a \sin \alpha}{r \cos \theta-a \cos \alpha}\right)-\tan ^{-1}\left(\frac{r \sin \theta+a \sin \alpha}{r \cos \theta-a \cos \alpha}\right)\right\}+\frac{1}{2} \operatorname{mar}\left(1-\frac{r^{2}}{a^{2}}\right) \\
\left\{\frac{\sin (\theta+\alpha)}{\left(r^{2}+a^{2}-2 r a \cos (\theta+\alpha)\right)}-\frac{\sin (\theta-\alpha)}{\left(r^{2}+a^{2}-2 r a \cos (\theta-\alpha)\right)}\right\}
\end{gathered}
$$

which is in agreement with that obtained by Ranger (1961) by a different method. The above stream function $\psi_{1}(r, \theta)$ can be expressed in the form

$$
\begin{aligned}
& \psi_{1}(r, \theta)=-2 m \alpha+2 m \alpha \sum_{n=1}^{\infty} f_{n}(r) \cos n \theta \\
& \text { where } f_{n}(r)=-2 m\left(\frac{r}{a}\right)^{n}\left\{\frac{1}{n}+\frac{1}{2}\left(1-\frac{r^{2}}{a^{2}}\right)\right\} \sin n \alpha
\end{aligned}
$$

(b) Flow between two co-axial circular cylinders, inner one being shear-free and outer one rigid

Now we place a shear-free circular cylinder of radius $b(<a)$ in a co-axial position with the outer cylinder.

The stream function for the resulting motion may be given by

$\psi_{3}=\psi_{1}+\psi_{2}$

where $\psi_{1}$ is the stream function (3) and $\psi_{2}$ is an appropriate general solution of (7) given by

$$
\psi_{2}=\sum_{n=1}^{\infty} g_{n}(r) \cos n \theta
$$

in which

$$
\text { and } \left.\begin{array}{c}
g_{n}(r)=A_{n} r^{n}+\frac{B_{n}}{r^{n}}+C_{n} r^{n+2}+\frac{D_{n}}{r^{n-2}} \quad(n>1), \\
g_{1}(r)=A_{1} r+B_{1} r^{3}+\frac{C_{1}}{r}+D_{1} r \ln r,
\end{array}\right\}
$$

$A_{n}, B_{n}$, and $D_{n}(n=1,2,3, \ldots)$ are arbitrary real coefficients.

The stream function $\psi_{1}$ in (13) satisfies the conditions fluid motion inside the boundary $r=a$; therefore, the velocity components derived from stream function $\psi_{2}$ only need to vanish on the outer cylinder $r=a$, implying that

$$
\psi_{2}=0=\frac{\partial \psi_{2}}{\partial r} \quad \text { on } \quad r=a
$$

Again, the normal velocity vanishes on the inner cylinder $r=b$, implying that 
$\psi_{3}=0 \quad$ on $\quad r=b$.

The shear-free condition on the inner cylinder may be derived as follows; the tangential stress component in plane polar coordinates $(r, \theta)$ is

$$
\tau_{r \theta}=\mu\left[\frac{1}{r} \frac{\partial u_{r}}{\partial \theta}+r \frac{\partial}{\partial r}\left(\frac{u_{\theta}}{r}\right)\right]=\mu\left[\frac{1}{r} \frac{\partial^{2} \psi_{3}}{\partial \theta^{2}}+r \frac{\partial}{\partial r}\left(\frac{1}{r} \frac{\partial \psi_{3}}{\partial r}\right)\right]
$$

where $\mu$ is the dynamic viscosity; thus, boundary condition $\tau_{r \theta}=0 r=b$ leads to

$$
\frac{\partial}{\partial r}\left(\frac{1}{2} \frac{\partial \psi_{3}}{\partial r}\right)=0 \quad \text { on } r=b
$$

Substitutions of equations (14) and (13) into equations (16), (17) and (19) respectively yield

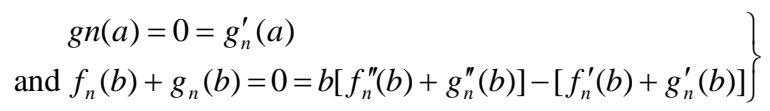

where the dashes denote differentiations with respect to $r$.

Equation (20) constitutes the following simultaneous linear equations to determine the constants $A_{n}, B_{n}, C_{n}$ and $D_{n}$.

For $n>1$.

$A_{n} a^{n}+\frac{B_{n}}{a^{n}}+C_{n} a^{n+2}+\frac{D_{n}}{a^{n-2}}=1$,

$n A_{n} a^{n}-n \frac{B_{n}}{a^{n}}+(n+2) C_{n} a^{n+2}-(\mathrm{n}-2) \frac{D_{n}}{a^{n-2}}=0$,

$A_{n} b^{n}+\frac{B_{n}}{b^{n}}+C_{n} b^{n+2}+\frac{D_{n}}{b^{n-2}}=-f_{n}(b)$,

$n(n-2) A_{n} b^{n-1}+n(n+2) \frac{B_{n}}{b^{n+1}}+n(n+2) C_{n} b^{n+1}+n(n-2) \frac{D_{n}}{b^{n-1}}=-\left[b f_{n}^{\prime \prime}(b)-f_{n}^{\prime}(b)\right]$

and for $n=1$,

$A_{1} a+B_{1} a^{3}+\frac{C_{1}}{a}+D_{1} a \ln a=0$,

$A_{1} a+3 B_{1} a^{3}+\frac{C_{1}}{a}+D_{1} a(1+\ln a)=0$,

$A_{1} b+B_{1} b^{3}+\frac{C_{1}}{a}+D_{1} b \ln b=-f_{1}(b)$,

$-A_{1}+3 B_{1} b^{2}+3 \frac{C_{1}}{b^{2}}-D_{1} \ln b=-\left[b f_{1}^{\prime}(b)-f_{1}^{\prime}(b)\right]$ 
Solving we obtain,

$$
\begin{aligned}
& K_{n} A_{n}=-m\left(\frac{b}{a}\right)^{n} \sin n \alpha a^{2} b^{n}\left[n(n+2) a^{2 n+2}-n(n+2) a^{2 n} b^{2}+(n+2) b^{2 n+2}-n a^{2 n-2} b^{4}\right] \\
& K_{n} B_{n}=-m\left(\frac{b}{a}\right)^{n} \sin n \alpha a^{2 n} b^{n+2}\left[n a^{2 n} b^{2}+(n+2) a^{2} b^{2 n}\right] \\
& K_{n} C_{n}=m\left(\frac{b}{a}\right)^{n} \sin n \alpha b^{n}\left[n(n+2)(n-1) a^{2 n+2}-n^{2} a^{2 n-2} b^{4}+n b^{2 n+2}\right] \\
& K_{n} D_{n}=m\left(\frac{b}{a}\right)^{n} \sin n \alpha a^{2 n} b^{n}\left[(n+2) a^{2 n+2}+n b^{2 n+2}\right]
\end{aligned}
$$

where $K_{n}=n a^{2} b^{2}\left[\left(a^{4 n}-b^{4 n}\right)-n a^{2 n-2} b^{2 n-2}\left(a^{4}-b^{4}\right)\right]$,

for $n=1, A_{1}, B_{1}, C_{1}$ and $D_{1}$ are given by

$$
\begin{aligned}
K_{1} A_{1} & =a m \sin \alpha\left[3\left(a^{4}-b^{4}\right)-6\left(a^{4}+b^{4}\right) \ln a+4 b^{4} \ln b\right], \\
K_{1} B_{1} & =-\frac{1}{a} m \sin \alpha\left[3 a^{4}-b^{4}-2 b^{4} \ln \left(\frac{a}{b}\right)\right], \\
K_{1} C_{1} & =2 m \sin \alpha a^{3} b^{4}\left[1+\ln \left(\frac{a}{b}\right)\right], \\
K_{1} C_{1} & =2 m \sin \alpha\left[3 a^{4}+b^{4}\right], \\
\text { where } K_{1} & =a^{2}\left[\left(a^{4}-b^{4}\right)-2\left(a^{4}+b^{4}\right) \ln \left(\frac{a}{b}\right)\right]
\end{aligned}
$$

Thus the function (15) is explicitly determined, and the stream function (13) for the viscous fluid motion between the cylinders appears as

$$
\begin{gathered}
\psi_{3}=-2 m \alpha+\sum_{n=1}^{\infty}\left(f_{n}(r)+g_{n}(r)\right) \cos n \theta \\
=-2 m \alpha+\sum_{n=1}^{\infty}\left[-2 m\left(\frac{r}{a}\right)^{n}\left\{\frac{1}{n}+\frac{1}{2}\left(1-\frac{r^{2}}{a^{2}}\right)\right\} \sin n \alpha\right] \cos n \theta+\left(A_{1} r+B_{1} r^{3}+\frac{C_{1}}{r}+D_{1} r \ln r\right) \\
\cos \theta+\sum_{n=2}^{\infty}\left(A_{n} r^{n}+\frac{B_{n}}{r^{n}}+C_{n} r^{n+2}+\frac{D_{n}}{r^{n-2}}\right) \cos n \theta
\end{gathered}
$$

where the values of the constants $A_{1}, B_{1}, C_{1}$ and $D_{1}$ and $A_{n}, B_{n}, C_{n}$ and $D_{n}$ being given by the relations (29) to (37). 


\section{CALCULATION OF THE FORCE AND TORQUE ON THE INNER CYLINDER}

In this section the authors derived formulae for the components of hydrodynamic force $(X, Y)$ and the torque $T$ on the inner cylinder $r=b$ in terms of stream function $\psi_{3}$.

$$
\left.\begin{array}{l}
X=\int_{0}^{2 \pi}\left(\tau_{r r} \cos \theta-\tau_{r \theta} \sin \theta\right)_{r=b} b d \theta, \\
Y=\int_{0}^{2 \pi}\left(\tau_{r r} \sin \theta+\tau_{r \theta} \cos \theta\right)_{r=b} b d \theta, \\
T=\int_{0}^{2 \pi}\left(\left(\tau_{r r} \cos \theta+\tau_{r \theta} \sin \theta\right) b \sin \theta+\left(\tau_{r r} \sin \theta+\tau_{r \theta} \cos \theta\right) b \cos \theta\right)_{r=b} b d \theta
\end{array}\right\}
$$

where

$$
\left.\begin{array}{rl}
\tau_{r r} & =-p+2 \mu \frac{\partial u_{r}}{\partial r}, \\
\text { and } & t_{r \theta}=\mu\left(\frac{1}{r} \frac{\partial u_{r}}{\partial r}+r \frac{\partial}{\partial r}\left(\frac{u_{\theta}}{r}\right)\right)
\end{array}\right\},
$$

are the components of the stress tensor $\tau_{r r}$; we note that $p$ is the fluid pressure and $\mu$ is the dynamic viscosity. And it is obtained

$$
\left.\begin{array}{l}
\tau_{r r}=-p, \\
t_{r \theta}=-\mu \frac{\partial^{2} \psi_{3}}{\partial r^{2}}
\end{array}\right\} \text { on } r=b .
$$

The equations of motion referred to plane polar coordinates $(r, \theta)$ may be written as

$$
\frac{\partial p}{\partial r}=\mu \frac{1}{r} \frac{\partial}{\partial \theta}\left(\nabla^{2} \psi_{3}\right),
$$

and $\frac{1}{r} \frac{\partial p}{\partial \theta}=-\mu \frac{\partial}{\partial r}\left(\nabla^{2} \psi_{3}\right)$

where $\nabla^{2} \equiv\left(\frac{\partial^{2}}{\partial r^{2}}+\frac{1}{r} \frac{\partial}{\partial r}+\frac{1}{r^{2}} \frac{\partial^{2}}{\partial \theta^{2}}\right)$.

Substitution of the results (41) and the equations (42) and (43) into (39) leads to the following formulae

$$
\left.\begin{array}{l}
X=\mu b^{2} \int_{0}^{2 \pi}\left(\frac{\partial^{3} \psi_{3}}{\partial r^{3}}\right)_{r=b} \sin \theta d \theta, \\
Y=\mu b^{2} \int_{0}^{2 \pi}\left(\frac{\partial^{3} \psi_{3}}{\partial r^{3}}\right)_{r=b} \cos \theta d \theta, \\
\text { and } T=\mu b^{2} \int_{0}^{2 \pi}\left(\frac{\partial^{2} \psi_{3}}{\partial r^{2}}\right)_{r=b} d \theta
\end{array}\right\}
$$


Substitution of (38) into (44) yields

$$
\begin{aligned}
& X=\mu b^{2} \sum_{n=1}^{\infty}\left[f_{n}^{\prime \prime \prime}(b)+g_{n}^{\prime \prime \prime}(b)\right] \int_{0}^{2 \pi} \cos n \theta \sin \theta d \theta \\
& Y=\mu b^{2} \sum_{n=1}^{\infty}\left[f_{n}^{\prime \prime \prime}(b)+g_{n}^{\prime \prime \prime}(b)\right] \int_{0}^{2 \pi} \cos n \theta \sin \theta d \theta \\
& \text { and } Y=\mu b^{2} \sum_{n=1}^{\infty}\left[f_{n}^{\prime \prime}(b)+g_{n}^{\prime \prime}(b)\right] \int_{0}^{2 \pi} \cos n \theta d \theta
\end{aligned}
$$

The evaluation of the force components and of the torque depends on $\int_{0}^{2 \pi} \cos n \theta \sin \theta d \theta, \quad \int_{0}^{2 \pi} \cos n \theta \cos \theta d \theta, \quad$ and $\int_{0}^{2 \pi} \cos n \theta d \theta$.

The first integral is zero for all positive integers, and the second one is zero for $5 \geq 2$ and equals to $\pi$ for $n=1$ and the last one is also zero.

Thus $X=0, Y=-\pi \mu b^{2}\left[f_{1}^{\prime \prime \prime}(b)+g_{1}^{\prime \prime \prime}(b)\right]$ and $T=0$.

The force $\mathrm{Y}$ on calculation appears as

$$
Y=2 \pi m \mu\left[\frac{12 a^{2} b^{2}+3 a^{4}+b^{4}+12 a^{2} b^{2} \ln \left(\frac{a}{b}\right)}{a\left\{\left(a^{4}-b^{4}\right)-2\left(a^{4}+b^{4}\right) \ln \left(\frac{a}{b}\right)\right\}}\right] \sin \alpha, a>b .
$$

Clearly the expression within the square bracket in (49) is negative, noting that $\alpha$ is within the interval $0<\alpha<\frac{\pi}{2}$. This indicates that the total force per unit length on the inner cylinder is in the same sense of the direction of the flow the source to the sink.

\section{RESULTS AND DISCUSSION}

It is interesting to see the variation of the force $Y$ with the radius of the inner shearfree circular boundary keeping the outer boundary fixed with constant radius unity.

Table 1. Numerical values of the force on the inner shear-free cylinder, where the outer one is rigid.

\begin{tabular}{cccccccccccc}
\hline $\mathrm{b}$ & 0 & 0.1 & 0.2 & 0.3 & 0.4 & 0.5 & 0.6 & 0.7 & 0.8 & 0.9 & 1 \\
\hline$\frac{-y}{4 \pi m \mu \sin \alpha}$ & 0 & 0.4710 & 0.9557 & 1.88 & 3.70 & 7.60 & 17.02 & 44.97 & 165.48 & 1418.71 & $\infty$ \\
\hline
\end{tabular}

Here it is important to note that Table 1 is for the case where inner cylinder is shearfree and outer one is rigid. Table 2 shows the numerical values of the force corresponding to those in table 1, obtained by using Ranger's formula (Ranger 1961) for the case where both the cylinders are rigid. 
Table 2. Numerical values of the force on the inner rigid cylinder, where the outer one is also rigid.

\begin{tabular}{cccccccccccc}
\hline $\mathrm{b}$ & 0 & 0.1 & 0.2 & 0.3 & 0.4 & 0.5 & 0.6 & 0.7 & 0.8 & 0.9 & 1 \\
\hline$\frac{-y}{4 \pi m \mu \sin \alpha}$ & 0 & 2.25 & 4.26 & 7.68 & 14.18 & 27.91 & 61.40 & 162.74 & 611.21 & 5423.23 & $\infty$ \\
\hline
\end{tabular}

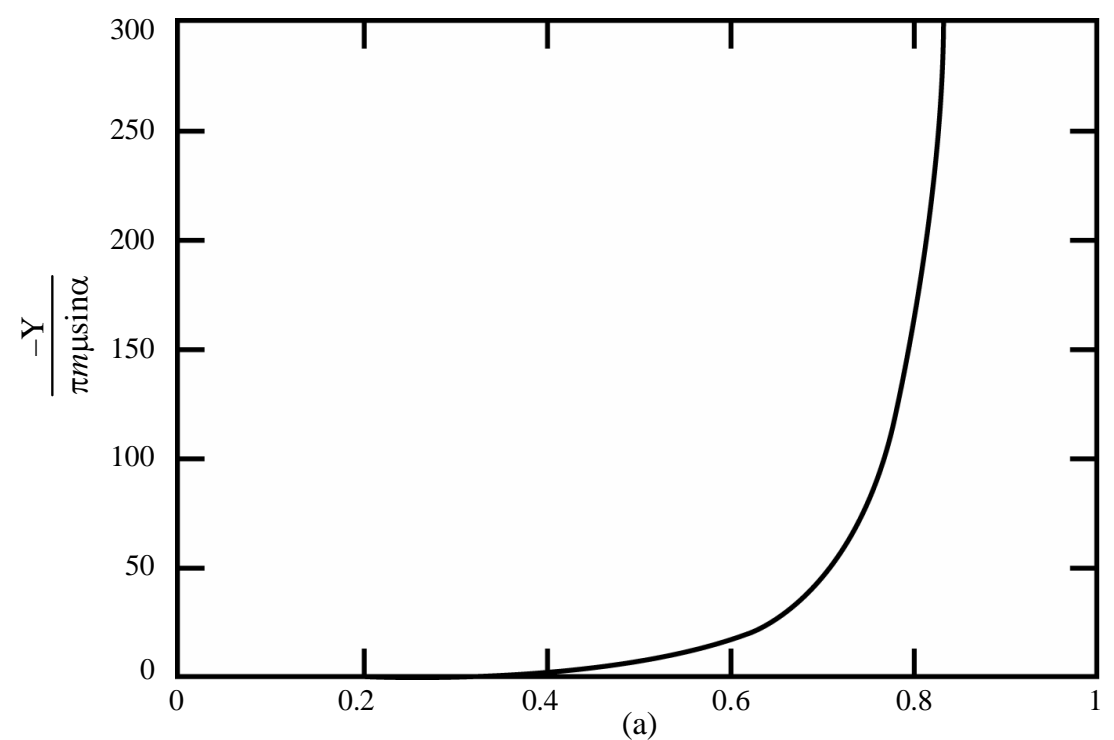

Fig. 2(a). Variation of the force on the inner shear-free cylinder with its radius, the outer one being rigid.

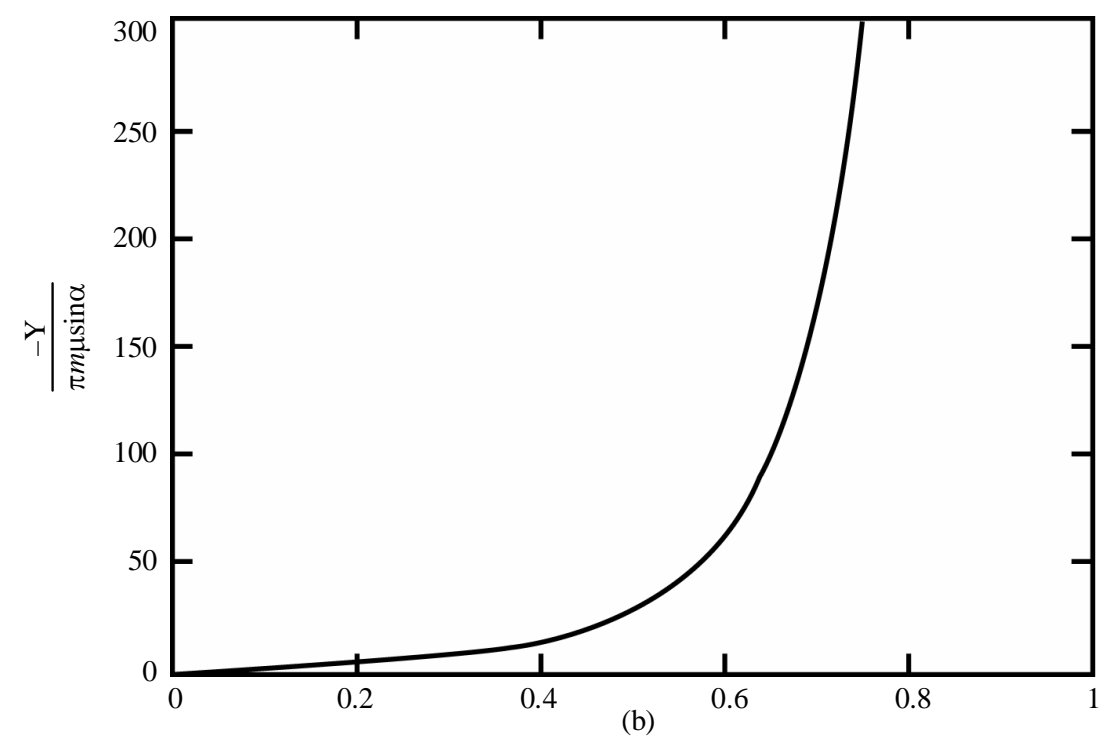

Fig. 2(b). Variation of the force on the inner rigid cylinder with its radius, the outer one being rigid. 
Fig. 2(a, b) depict the variation of the force with the radius of the inner circular cylinder for the cases: (i) The inner cylinder is shear-stress-free where the outer one is rigid; and (ii) both the cylinders are rigid. It is seen from the Fig. 2(a), or the case of shear-stresstree inner cylinder and the outer rigid one, the force on the inner cylinder with the increase of its radius, begins from very small value, initially increases very slowly and this trend continues until the radius of the inner cylinder exceeds some value around 0.6; after crossing this value the force increases relatively rapidly. When the value of the radius of the inner cylinder approaches unity the force increases beyond limit. Again it is seen from the Fig. 2(b), for the case of rigid cylinders, that the force on the inner cylinder initially increases slowly and very rapidly; finally, the force approaches infinity as the radius approaches unity.

\section{APPENDIX}

It is to establish a circle theorem for plane Stokes flows which will help to obtain the resulting flow within the circular boundary due to the introduction of a rigid circular cylinder, with small perforations which facilitate placement of singularities therein, the basic flow being due to the same singularities. The theorem provides an expression for the perturbed stream function $\psi_{0}^{*}$ in terms of the basic stream function $\psi_{0}$.

The circle theorem: Let there be a slow and irrigational two-dimensional flow of an incompressible viscous fluid in the absence of rigid boundaries and let the flow be characterized by the stream function $\psi_{0}=\psi_{0}(r, \theta)$ where the singularities are all at a distance a from the origin and let $\psi_{0} \sim 0\left(r^{k}\right), k>1$ at the origin and $\psi_{0}(r, \theta)=0$. If now a circular cylinder $r=a$, with perforations on it is introduced into the flow to allow the singularities to stay therein the stream function for the resulting flow inside the cylinder becomes

$$
\psi(r, \theta)=\psi_{0}(r, \theta)+\frac{\left(a^{2}-r^{2}\right) r}{2 a^{2}} \frac{\partial \psi_{0}(r, \theta)}{\partial r} .
$$

For the purpose of the proof of the theorem the following lemma is needed:

Lemma. If $\psi=\psi(r, \theta)$ satisfies the Laplace's equation $\nabla^{2} \psi=0$, then $r \frac{\partial \psi(r, \theta)}{\partial d}$ satisfies the same equation.

\section{Proof of the lemma:}

Since $\nabla^{2} \psi(r, \theta)=0$,

then $\nabla^{2}\left(r \frac{\partial \psi(r, \theta)}{\partial r}\right) 2 \nabla^{2} \psi(r, \theta)+r \frac{\partial}{\partial r}\left(\nabla^{2} \psi(r, \theta)\right)=0$. 
Thus the lemma is proved.

Proof of the circle theorem: As the flow is irrotational, we have

$\nabla^{2} \psi(r, \theta)=0$.

Therefore, by the lemma, $\nabla^{2}\left(r \frac{\partial \psi_{0}(r, \theta)}{\partial r}\right)=0$.

As all harmonic functions are bi-harmonic functions; therefore, $r \frac{\partial \psi_{0}(r, \theta)}{\partial r}$ is a biharmonic function.

From Bramble (1958), it is known that if $\psi=\psi(r, \theta)$ is a harmonic function, then $r^{2} \psi(r, \theta)$ is a bi-harmonic function.

Therefore, $r^{2}\left(r \frac{\partial \psi_{0}(r, \theta)}{\partial r}\right)$ is a bi-harmonic function.

Therefore, as a whole $\frac{\left(a^{2}-r^{2}\right)}{2 a^{2}} \frac{\partial \psi_{0}(r, \theta)}{\partial r}$ is a bi-harmonic function.

The stream function (1) clearly shows that

$\psi(r, \theta)=0$ on $r=a$,

on noting the condition $\psi(r, \theta)=0$.

Now,

$$
\begin{aligned}
& \frac{\partial \psi(r, \theta)}{\partial r}=\frac{\partial \psi_{0}(r, \theta)}{\partial r}-\frac{r^{2}}{a^{2}} \frac{\partial \psi_{0}(r, \theta)}{\partial r}+\frac{\left(a^{2}-r^{2}\right)}{2 a} \frac{\partial \psi_{0}(r, \theta)}{\partial r}+\frac{\left(a^{2}-r^{2}\right) r}{2 a^{2}} \frac{\partial^{2} \psi_{0}(r, \theta)}{\partial r^{2}} \\
& \text { Clearly } \frac{\partial \psi(r, \theta)}{\partial r}=0 \text { on } r=a .
\end{aligned}
$$

Thus (1) satisfies all the conditions and is therefore the stream function of the resulting motion. Hence the theorem is proved.

\section{REFERENCES}

Bramble, J.H. 1958. Continuation of biharmonic functions across circular arcs. J. Math. Mech. 7(6): 904-924.

Chen, O.X. 2000. Two-dimensional flow through an annular junction. Can. Appl. Math. Q. 8(3): 203-214.

Daripa, P. and Palaniappan, D. 2001. Generalized circle and sphere theorems for inviscid and viscous flows SIAMJ. Appl. Math. 62(2): 514-540.

Figueroa-Espinoza, B., Zenit, R. and Legendre, D. 2008. The effect of confinement on the motion of a single clean bubble. J. Fluid Mech. 616: 419-443.

Harper, J. and Brenner, H. 1983. Low Reynolds number hydrodynamics. Second edition, martinus Nijhoff Publishers, Dordrecht, pp. 130-133,321,387-399,448-46. 
Harper, J.F. 1983. Axisymmetric Stokes flow images in spherical free surfaces with application to rising bubbles. J. Austral. Math. Soc. Ser. B. 25: 217-231.

Legendre, D., Magnaudet, J. and Mougin, G. 2003. Hydrodynamic i8nteractions between two spherical bubbles rising side by side in a viscous liquid. J. Fluid Mech. 497: 133-166

Palaniappan, D., Nigam, S.D. and Amaranath, T. 1996. Shear-free boundary in Stokes flow. Internat. J. Math. Sct. 19(1): 145-150.

Palaniappan, D., Nigam, S.D., Amaranath, T, and Usha, R. 1990. A theorem for a shear free sphere in Stokes flow. Mech. Res. Comm. 17: 429-435.

Ranger, K.B. 1961. A problem on the slow motion of a viscous fluid between two fixed cylinders Q.J. Mech. Appl. Math. 14(4): 411-421.

Rayleigh, Lord 1893. On the flow of viscous liquids, especially in two dimensions. Phil. Mag. 36: 354-372; Scientific Papers 4: 78.

Usha, R. and Hemalatha, K. 1993. A note on plane Stokes flow past a shear free impermeable cylinder. Z. Angew. Math. Phys. (ZAMP) 44: 73-84.

(Received revised manuscript on 7 February, 2012) 